\title{
Canonicidad y complejidad en el sistema verbal del español: implicaciones para los procesos de enseñanza/aprendizaje en español
}

\section{Canonicity and complexity in the Spanish verbal system: implications for teaching/learning processes}

\section{Théophile Ambadiang Omengele}

Universidad Autónoma de Madrid

theophile.ambadiang@uam.es

ORCID ID: https://orcid.org/0000-0002-2204-2506

DOI: $10.17398 / 209 / 1988-8430.35 .1 .209$

Esta obra está publicada bajo una licencia Creative Commons

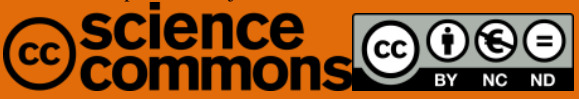

Fecha de recepción: 30/04/2021 Fecha de aceptación: 18/05/2021

OPEN ACCESS

Ambadiang Omengele, T. (2022). Canonicidad y complejidad en el sistema verbal del español: implicaciones para los procesos de enseñanza/aprendizaje en español. Tejuelo, 35(1), 209-240. Doi: https://doi.org/10.17398/1988-8430.35.1.209 
Resumen: Los sistemas flexivos presentan, entre otras características, un grado variable de congruencia, al resultar su configuración interna de la tensión que se produce entre cierta tendencia a la complejidad, asociada a la ausencia de congruencia interna, y a la sistematicidad, entendida en términos de canonicidad. Por otra parte, los usuarios de una lengua, y particularmente los aprendices, tienden a interpretar su sistema flexivo de acuerdo con unas pautas de congruencia que pueden no solo resultar diferentes de las que se consideran propias de dicho sistema, sino incluso tener el efecto de incrementar su complejidad. Intentamos mostrar que una caracterización basada en estas características permite una representación más simple de los paradigmas de los lexemas verbales, al requerir solo aquellas formas que resultan impredecibles ('formas de referencia', Stump y Finkel 2013, 2015). Estas últimas entran en dos tipos de relaciones: relaciones de doble implicación o simétricas entre ellas, ligadas a afinidades de carácter procesual, y relaciones de implicación o asimétricas con las formas predecibles, asociadas a afinidades formales. Cotejamos las implicaciones de este tipo de representación con otras basadas exclusivamente en afinidades formales $y$ en unidades subléxicas y resaltamos el interés teórico y pedagógico de generalizaciones basadas en el concepto de 'forma de referencia'.

Palabras clave: flexión verbal; canonicidad; complejidad; aprendizaje; formas de referencia.
Abstract: Inflectional systems present, among other characteristics, a variable degree of internal congruence, as their internal configuration results from the tension that occurs between a certain tendency to complexity, associated with the absence of internal congruence, and to systematicity, understood in terms of canonicity. On the other hand, users of a language, and particularly learners, tend to interpret its inflectional system according to congruence patterns that may not only be different from those considered proper to that system, but even have the effect of increase its complexity.

We show that a characterization based on such features allows a simpler representation of the paradigms of the verbal lexemes, by requiring only those forms that are unpredictable ('principal parts', Stump \& Finkel 2013, 2015). Such forms fall into two types of relationships: doubly implicational or symmetrical relationships between them, linked to processual affinities, and implicational or asymmetrical relationships with predictable forms, associated with formal affinities. We compare the implications of this type of representation with others based exclusively on formal affinities and sublexical units, and we highlight the theoretical and pedagogical interest of generalizations based on the concept of 'principal part'.

Keywords: verbal inflection; canonicity; complexity; learning; principal parts. 


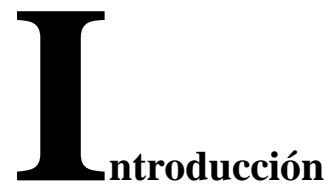

Si bien en general la regularidad de un sistema está ligada de manera característica a su transparencia y simplicidad y, por tanto, se puede interpretar como una manifestación de la 'canonicidad' entendida en términos de lo que 'debería haber' o "lo que debería ser", mientras que la irregularidad está ligada a la opacidad y a la complejidad entendida como lo que hay o lo que es el sistema considerado en sí mismo (cf. Corbett, 2007; 2009; Brown y Chumakina, 2013; Anderson, 2015), en la práctica coexisten en los sistemas flexivos de las diferentes lenguas, si bien su interrelación no tiene necesariamente las mismas implicaciones (Ackerman et al., 2009; Stump y Finkel, 2013, Ackerman y Malouf, 2016). En este trabajo nos proponemos estudiar la manera en que la tensión que se produce entre la tendencia a la regularidad e irregularidad (así como en los elementos asociados a ellas) incide en la configuración interna del sistema verbal del español desde el punto de vista de los procesos de enseñanza/aprendizaje. El punto de partida es que las producciones de los hablantes y, sobre todo, de los aprendices del español muestran que tienden a limitar o reducir los efectos de la complejidad inherente en el sistema verbal, al postular la existencia de relaciones formales y/o funcionales entre las formas verbales $\mathrm{y}$, sobre esta base, una distribución paradigmática de las mismas que consideran 
más coherente, lo que supone preferir cierta uniformidad y congruencia a alternativas que entrañarían una mayor complejidad.

Las producciones de los aprendices sugieren que muchas de esas relaciones son de implicación y que tienden a basarse en factores que tienen que ver con las características formales de los elementos que conforman cada paradigma verbal. Teniendo en cuenta esta observación, empezamos con una breve reseña de descripciones recientes del sistema verbal del español que postulan relaciones que se pueden interpretar como de implicación, con el objeto de intentar una explicitación de las clases de relaciones que se producen en este sistema verbal. Este intento da pie a la propuesta de representación léxica de los lexemas verbales que se hace en el penúltimo apartado. Las formas involucradas en la representación léxica de un lexema verbal, generalmente un subconjunto de los elementos que conforman su paradigma, desempeñan el papel de 'formas de referencia' (principal parts, Finkel y Stump, 2007, 2009; Stump y Finkel, 2013, 2015; Ackerman, F., J.P. Blevins y R. Malouf; Ackerman y Malouf, 2016), en el sentido de que, además de ser básicas e impredecibles en comparación con las formas restantes, determinan de algún modo su distribución (además de sus características formales). Desde este punto de vista, se pueden considerar como indicadores de la congruencia interna del sistema verbal $\mathrm{y}$, como tales, son formas y puntos de referencia que resultan cruciales para los procesos de enseñanza/aprendizaje del sistema verbal español, como sugiere la propuesta didáctica esbozada en el penúltimo apartado.

\section{1.- Canonicidad y flexión regular}

En términos generales, los fenómenos flexivos se tienden a caracterizar como regulares e irregulares. La presencia de fenómenos irregulares dentro del paradigma de un lexema tiende a inducir a considerarlo como irregular. Con todo, esta manera de caracterizar la irregularidad, asociada a variaciones formales que se producen en la raíz, el tema y/o la desinencia de una forma determinada y que requieren reglas o procesos específicos en el sistema morfológico 
considerado o bien la inclusión de información específica en el lexicón (Sagot y Walther, 2011: 26), no permite dar cuenta de todos los desajustes existentes entre forma y contenido, asociado este último a la función paradigmática (Stump, 2001). A modo de ejemplo, no permitiría tratar de manera interesante la diferencia que se produce entre, por un lado, los paradigmas de los verbos regulares de primera y tercera conjugaciones, cuyas formas de primera persona de plural del presente de indicativo y pretérito indefinido (cf. cantamos, vivimos) son idénticas y, por otro, el de los verbos regulares de segunda conjugación, cuyas formas de primera persona de plural del presente de indicativo y del pretérito indefinido difieren (cf. comemos, comimos). Uno de los efectos de esta diferencia es que los paradigmas de los verbos de la segunda conjugación presentan un menor grado de complejidad que los de los verbos de la primera y tercera conjugaciones, en el supuesto de que la presencia de formas sincréticas el paradigma de un verbo tiene el efecto de incrementar lo que se podría definir como su complejidad descriptiva (en contraposición con la complejidad típica de los modelos teóricos; cf. Sagot y Walther, 2011), en proporción con los casos en que la relación entre elementos de forma y de contenido deja de ser 'uno-auno'. Si bien desde un punto de vista formal se suele dar cuenta de fenómenos de sincretismo como el señalado antes por medio de lo que serían reglas de remisión (rules of referral), el hecho de que la configuración formal de piezas léxicas como las mencionadas antes induce a interpretarlas como regulares da pie a que sean tratadas del mismo modo, a pesar de la diferencia que acabamos de señalar entre ellas. Diferencias de este tipo, en las que está implicada la estructura del paradigma de un lexema además de las formas que lo componen, entrañan cierto interés desde un punto de vista tanto descriptivo como teórico y pedagógico.

Consideraciones de este último tipo han inducido a algunos estudiosos a propugnar una caracterización más fina de la (ir)regularidad, en la que interviene no solo la configuración morfológica de las formas de cada lexema y el ajuste entre forma y función (o contenido), sino también otros factores, tales como la distribución de una forma completa dentro del paradigma de su lexema. De acuerdo con una caracterización de este tipo, ni siquiera los 
paradigmas de los verbos tradicionalmente considerados como regulares lo son del todo, al menos en una interpretación que asocia la regularidad con la ausencia de complejidad. Más aún, da pie para cuestionar las propuestas de caracterización de la regularidad y la irregularidad formuladas en términos estrictamente oposicionales. En efecto, ambos fenómenos pueden producirse en diferentes niveles y tener un alcance variado tanto dentro del paradigma de un lexema como en un sistema flexivo dado. El alcance de la (ir)regularidad dentro del paradigma de un lexema depende de su presencia en (cada una de) sus formas y en (cada uno de) los sub-paradigmas que lo componen, mientras que su alcance en un sistema flexivo depende del que tiene en los subparadigmas y los paradigmas de los diferentes lexemas.

Teniendo en cuenta estas últimas observaciones, los paradigmas y los sistemas flexivos pueden tipificarse sobre la base de un modelo teórico ideal, reflejo de cómo debería ser un paradigma o un sistema flexivo (Brown y Chumakina, 2013), y que serviría de referencia a la hora de determinar la anomalía o marginalidad de un paradigma o un sistema flexivo dado. Simplificando mucho, en referencia a las estructuras paradigmáticas, a ese ideal teórico se le asocian los rasgos siguientes (cf. Bond, 2019).

(1) Rasgos esenciales de un paradigma flexivo canónico

a. exhaustividad: el paradigma de un lexema presenta el mayor número de celdas posible, es decir, permite todas las combinaciones de rasgos morfosintácticos compatibles.

b. completitud: todas las celdas de un paradigma tienen cada una alguna celda asociada a ella.

c. especificidad: cada celda tiene asociada una forma específica, distinta de todas las demás-

Mientras que los rasgos incluidos en (1) remiten en una buena medida al ajuste de forma y función en las formas flexionadas incluidas en un paradigma, otros rasgos se añaden a ellos a la hora de caracterizar un sistema flexivo canónico. De acuerdo con Corbett (2007, 2009), la canonicidad de un sistema implica que sus formas presentan la misma configuración: su material léxico es variable para los diferentes lexemas, mientras que las desinencias varían en el paradigma de un 
lexema, pero son uniformes en los paradigmas de los diversos lexemas, de acuerdo con su clase flexiva en caso de darse esta distinción. Ello es consistente con la idea de la co-presencia dentro del sistema de formas flexionadas diferentes que ocupan cada una alguna de las celdas dentro del paradigma de cada lexema, es decir, para expresar cada una de las combinaciones de rasgos morfosintácticos.

Como pone de manifiesto esta caracterización, la regularidad tanto de una forma como de un paradigma o de un sistema flexivo se establece sobre la base de factores que, tomados de manera aislada, no se interpretan solo ni siempre en términos de regularidad, ni remiten necesariamente a ella. Más bien, remiten conjuntamente a la canonicidad que, si bien subsume a la regularidad, no tiene una relación directa con ella.

\section{2.- Flexión irregular y efecto de complejidad 2.1.- Irregularidad y efecto de complejidad}

A diferencia de lo que ocurre entre la regularidad y la canonicidad, existe una relación directa entre la complejidad de un paradigma o sistema flexivo y la cantidad y la distribución de las irregularidades que, como se ha señalado antes, se pueden asociar a reglas o procesos específicos dentro del sistema o bien a informaciones específicas dentro del lexicón (Sagot y Walther, 2011: 26). En términos generales, la determinación de la complejidad de un paradigma o de sistema flexivo pasa por el estudio de la configuración interna de las palabras y de las marcas morfológicas implicadas en su formación (Anderson, 2015) y, en último término, por la identificación de los procesos asociados a todas sus formas irregulares, es decir, de los fenómenos no canónicos. De acuerdo con Brown y Chumakina (2013), fenómenos de este tipo sirven para determinar la distancia relativa que existe entre un sistema o un paradigma flexivo concreto y el ideal canónico que le corresponde. Los fenómenos no canónicos más comunes están asociados a procesos tales como la suplencia (morfómica, morfosintáctica, parcial o bien total), la heteroclisis, la 
defectividad, la deponencia, la redundancia, así como el sincretismo en sus diferentes versiones.

\section{2.- Irregularidad y complejidad en el sistema verbal del español}

El sistema verbal del español presenta muchos de los fenómenos asociados a la irregularidad y los vamos a describir y ejemplificar de manera breve, empezando por la suplencia. Puesto que en general las formas verbales presentan algún tipo de desinencia, ni la relación de suplencia, ni la de alomorfia más generalmente, se aplican a formas verbales completas. Por el contrario, existen muchos casos de alomorfia y suplencia de raíz. Los casos de alomorfia están asociados a alternancias de variantes de raíz verbal estrechamente ligadas a tiempos verbales. Así, las variantes de raíz cuya vocal diptonga o sufre un proceso de cierre se usan de manera característica en las cuatro formas cortas del presente. Otra variación alomórfica típica del presente tiene que ver con alternancias que afectan a la configuración consonántica de la raíz en la forma de procesos de epéntesis (de /g, k/ en el presente y /d/

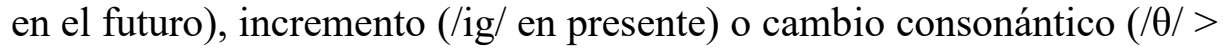
$/ \mathrm{g} /$ ) en el presente. La transparencia de la relación que existe entre las variantes implicadas en una relación de alomorfia entraña cierta variación, como sugiere la diferencia observada entre las variantes de raíz verbal típicamente usadas en el presente por un lado y, por otro, las variantes de raíz verbal presentes en los futuros irregulares y en los pretéritos indefinidos irregulares. Formas de esta última clase, que a menudo presentan procesos de alternancia mixta (vocálica y consonántica), se prestan más a una caracterización en términos de suplencia que las del presente e incluso que las del futuro.

Algunos lexemas se caracterizan porque son defectivos, al presentar sus paradigmas algunas celdas desprovistas de formas propias de esos lexemas. El alcance de esta característica tiende a variar de un verbo a otro, como muestra el cotejo de los paradigmas de verbos tales como abolir, garantir y desabrir, si dejamos de lado los llamados verbos impersonales. En contraposición con esta situación, los paradigmas de algunos verbos presentan celdas ocupadas por más de una forma. Al lado del caso típico de dobles participios pasados (cf. 
abstraído/abstracto, dividido/diviso, hartado/harto, imprimido/impreso, nacido/nato, soltado/suelto, teñidoltinto), y de dobletes acentuales en el presente (cf. adecúoladecuo) los paradigmas de los pocos verbos de doble infinitivo presentan dobletes (formas típicas de dos conjugaciones diferentes) en la mayor parte de sus celdas (cf. balbucear/balbucir, colorear/colorir). Si bien en el caso de estos verbos, cabe hablar de paradigmas diferentes e incluso de lexemas distintos, el hecho de que tienden a compartir entrada en los diccionarios con la indicación, a menudo, de que uno de ellos es poco usado o anticuado, da pie para que se les considere como variantes de un mismo lexema, con la salvedad de que la variación tiende a tener alcance sobre todo el paradigma en el sentido de que, en rigor, tendríamos dos paradigmas distintos (asociados a diferencias de conjugación en el caso de los lexemas mencionados) que funcionan de manera más o menos paralela, mientras que la relación de alomorfia se caracteriza en buena medida porque la distribución de las variantes de la raíz de cada verbo obedece a lo que se podría caracterizar como una relación complementaria.

Por último, existen sub-paradigmas temporales cuyas formas se caracterizan porque presentan raíces específicas (generalmente supletivas debido a procesos de alternancia mixta), cuya irregularidad las excluye de los espacios paradigmáticos que corresponden de manera típica a las tres conjugaciones (cf. anduv-, pus- tuv-, vin-, etc.) y desinencias que se pueden considerar como típicas de la primera conjugación en el caso de la primera y tercera persona del singular y de la segunda y tercera conjugaciones en el resto de formas, si consideramos el acento como un rasgo paradigmático (y solo de manera secundaria una característica de cada forma verbal considerada de manera aislada; cf. Ambadiang, 2017). De acuerdo con esta caracterización, los sub-paradigmas temporales típicos de los llamados pretéritos fuertes se pueden considerar como casos de heteroclisis (Stump y Finkel, 2013). En contraposición con esta marcada tendencia a la especificidad, los paradigmas verbales incluyen de manera característica celdas cuyas formas resultan idénticas. Coincidencias de este tipo, asociadas a relaciones sincréticas, se observan generalmente entre la primera y la tercera persona de singular de los sub-paradigmas del imperfecto de indicativo (cantaba, comía, vivía), así como en todos 
los sub-paradigmas del modo subjuntivo (cf. cante, cantase, cantare; coma, comiese, comiere; viva, viviese, viviere) y también entre las formas de primera persona del plural del presente de indicativo y del indefinido de los verbos regulares de primera y tercera conjugaciones, como se ha señalado antes. Las formas de primera y tercera persona de los pretéritos fuertes incrementan la complejidad característica de este sub-paradigma, al presentar una acentuación no paradigmática, al igual que ocurre con aquellas formas cortas del presente cuya acentuación no se puede predecir (cf. aíslo, confío, etc.). Igual ocurre con los dobletes acentuales en esa misma clase de formas (cf. adecúo/adecuo), así como en los casos de dobles participios (cf. imprimido/impreso) y de raíces supletivas (cf. ser/ir: fui), además de los sincretismos subparadigmáticos, como el que se produce en el indefinido entre estos dos verbos.

\section{3.- La congruencia paradigmática y su formalización}

El estudio de la canonicidad de los sistemas morfológicos subsume el de la complejidad paradigmática que, según se ha señalado antes, depende de las propiedades morfosintácticas, las variantes alomórficas implicadas en su materialización en formas verbales, así como de las conjugaciones en las que quedan distribuidas estas últimas en una lengua dada. Si bien en términos generales, las indagaciones enfocadas en la complejidad de los sistemas morfológicos basan sus generalizaciones en las relaciones que contraen las formas flexionadas en las que se materializan los distintos lexemas, tienden a presentar diferencias en su alcance y en la manera en que dan cuenta de la congruencia interna de los sistemas flexivos, es decir, desde el punto de vista de las clases de relaciones que establecen entre las formas incluidas en estos sistemas. En lo que se refiere al alcance, algunas propuestas centran sus observaciones en una parte del sistema flexivo, mientras que otras estudian la organización interna de los sistemas morfológicos.

Las generalizaciones típicas de los enfoques de alcance parcial se basan en (i) relaciones morfómicas, asociadas a patrones de identidad 
o afinidad formal (cf. Aronoff, 1994; Round, 2015), (ii) relaciones de implicación entre patrones de alternancia en los temas verbales (cf. Palancar, 2019) y (iii) redes de convergencia de procesos (cf. Herce, 2020). Los análisis basados en el morfoma se aplican exclusivamente en aquellos casos en que las formas consideradas presentan similitudes cuya motivación se supone exclusivamente morfológica, algo que, en el caso del español, se limitaría a las relaciones que se producen, respectivamente, entre la forma de primera persona de singular del presente de indicativo y las del presente de subjuntivo, entre las formas cortas y las formas largas del presente, así como entre la forma de tercera persona de plural del indefinido y las formas del imperfecto de subjuntivo (cf. Maiden, 2004, 2018; O’Neill, 2014). Por su parte, las relaciones de implicación que postula Palancar se producen entre cuatro clases de temas irregulares y tienen el efecto de reducir el coste cognitivo de aquellas formas que son dependientes: la diptongación implica el cierre de la vocal de la raíz en las formas del presente, y la inserción de $/ \theta / \mathrm{y} / \mathrm{g} /$ en la primera persona del presente de indicativo implica la alomorfia de raíz típica de algunos pretéritos fuertes. Por último, las redes de convergencia que postula Herce dan cuenta del paralelismo que se produce entre determinadas irregularidades, como es el caso entre la primera persona de singular del presente de indicativo y las formas del futuro y condicional de algunos verbos.

En lo que se refiere a las relaciones subsumidas en las generalizaciones de estas propuestas, si bien tienden a tener un carácter implicativo, los datos no siempre se ajustan a esta interpretación, como ocurre con el llamado 'morfoma N', asociado al contraste que se produce entre las formas cortas y largas del presente. En el mismo sentido, el análisis de Palancar se basa en una simplificación excesiva de los datos. Aparte de los problemas asociados al concepto de tema tal como lo caracteriza, el hecho de que es necesaria la misma información léxica para dar cuenta de las clases de irregularidad que estudia pone en tela de juicio la diferencia que establece respecto de su coste cognitivo respectivo. A modo de ejemplo, la relación que establece entre la irregularidad asociada a la inserción de $/ \theta / \mathrm{y} / \mathrm{g} /$ en la primera persona de singular del presente de indicativo y la alomorfia de raíz típica de algunos pretéritos fuertes no se produce en todos los verbos (cf. caer: 
caigo, caî). Por último, la convergencia de procesos en la que se basa el análisis de Herce parece observarse más allá de los verbos en los que centra su estudio (poner, tener, venir), según sugiere el paralelismo observado entre otras irregularidades típicas de la primera persona del presente de indicativo y de las formas del futuro (sé-sabré, quepocabré, etc.; pero nazco-naceré).

En contraste con estas propuestas que acabamos de reseñar de manera muy somera, los enfoques que estudian los sistemas flexivos como totalidades se caracterizan porque basan sus generalizaciones en las palabras o formas en que se materializan los lexemas, las relaciones que existen entre ellas dentro de sus paradigmas respectivos y el papel de sus constituyentes. Esas relaciones se interpretan en términos de implicación (cf. Finkel y Stump, 2007, 2009; Stump y Finkel, 2013, 2015), o bien de manera más compleja (cf. Ackerman, Blevins y Malouf, 2009; Ackerman y Malouf, 2016). Las relaciones de implicación están estrechamente asociadas al concepto de 'formas de referencia' y a la idea de que, entre esas formas, que son impredecibles, y aquellas que se pueden deducir a partir de ellas, existe una relación de dependencia que tiene el efecto de reducir la entropía del sistema y, por tanto, reforzar su congruencia interna. Con todo, formalizaciones de este tipo no dan cuenta de toda la complejidad del sistema, al basarse en la idea de una afinidad formal muy marcada ligada a la identidad relativa a la raíz (o a la desinencia) de las formas consideradas en cada caso (Stump y Funkel, 2013, p.79). Desde este punto de vista, propuestas basadas en relaciones de interdependencia permiten una formalización más interesante de la congruencia de los sistemas flexivos, en la medida en que dan cuenta de las relaciones asimétricas, del tipo que acabamos de señalar, como de las relaciones simétricas, que se producen entre formas impredecibles (cf. Ackerman, Blevins y Malouf, 2009). Como intentamos mostrar en el próximo apartado, las divergencias observadas en las relaciones que se producen entre formas irregulares dentro de los paradigmas de los lexemas verbales del español apoyan un enfoque de este tipo, además de la necesidad de proceder sobre la base de cada lexema o, mejor, del espacio paradigmático que le corresponde, puesto que las relaciones de 
interdependencia pueden variar del paradigma de un lexema dado al de otro.

\section{4.- Congruencia y efecto de complejidad en el sistema verbal del español}

\section{1.- Congruencia y complejidad en las producciones de aprendices}

Las producciones típicas de los aprendices del español, como lengua materna (Clahsen, Aveledo y Roca, 2002; Aveledo, 2006; Oliva, Serrano, del Castillo e Iglesias, 2017) o no (Ambadiang y García Parejo, 1999; Ambadiang y Camus Bergareche, 2002), se caracterizan en general porque incluyen diferentes clases de formas anómalas que resultan, sin embargo, de un esfuerzo en el sentido de dar (más) congruencia al sistema verbal. Con todo, el efecto aparente de este esfuerzo tiende a suponer un incremento de la complejidad del sistema verbal, en la medida en que sus errores están asociados a informaciones específicas dentro del sistema morfológico y/o del léxico que, además de reflejar cómo sería el sistema verbal de acuerdo con el criterio de cada aprendiz, pueden entrar en contradicción con otras informaciones que corresponden a ese sistema tal como es. A modo de ejemplo, para dar cuenta de formas del tipo de andía, corré, duermías, tuví y veniré se requieren especificaciones de los lexemas respectivos que contradicen las que corresponden a las formas existentes, a saber, andaba, corrí, dormías, tuve y vendré.

Por otra parte, las producciones de los aprendices se caracterizan porque entrañan cierto grado de variación interna, sean de uno o varios sujetos. Ello implica que cada individuo organiza de manera diferente el sistema verbal, es decir, que recurre a diferentes elementos que sirven como (puntos de) referencia para la organización del sistema verbal tal y como la concibe. Tales elementos son aquellas formas verbales completas que le resultan más familiares a cada uno, y la variación está ligada al análisis que cada uno hace de ellas. En este sentido, sirven de referencia para los procesos de segmentación y recombinación que subyacen a sus producciones respectivas. 
En su inmensa mayoría, los errores típicos de los aprendices tanto nativos como no nativos resultan de desajustes en la combinación de raíces y desinencias, una asignación errónea del rasgo de conjugación a un lexema verbal dado, la distribución errónea de alguna variante de la raíz de un lexema verbal o de una desinencia verbal, la asociación errónea de una desinencia a una persona verbal o bien a causas multifactoriales, como se intenta mostrar en la tipificación siguiente, que se basa en datos relativos a aprendices tanto nativos (cf. Clahsen, Aveledo y Roca, 2002; Aveledo, 2006; Oliva, Serrano, del Castillo e Iglesias, 2017) como no nativos (cf. Ambadiang y García Parejo, 1999; Ambadiang y Camus Bergareche, 2002).

(2) Breve tipificación de los errores de aprendices

a. Errores asociados a la conjugación verbal

andía (andaba), volimos (volamos), dormaba (dormía), comé (comí), corré (corrió)

b. Errores asociados a la distribución de las variantes de la raíz duermías (dormías), empiezó (empezó), juegábamos (jugábamos), sueñé (soñé), haceré (haré), veniré (vendré), punieron (pusieron)

c. Errores asociados a la desinencia de persona podría (podrías), quieres (quiere), tienes (tiene)

d. Errores asociados a la regularización de la desinencia vinió (vino), tuví (tuve), ibían (iban)

e. Errores multifactoriales vestiba (vestía), queriba (quería)

De acuerdo con esta distribución, los errores de los aprendices sugieren que la información que sirve de referencia puede ser de naturaleza más o menos abstracta. Puede tratarse de un rasgo léxico, como el relativo a la conjugación (cf. (2a, d)), de rasgos léxicos asociados de manera estable a determinados elementos formales (raíces, como en (2b)) o bien de una pauta errónea relativa a la formación y/o distribución de alguna desinencia (cf. (2c, e)), es decir, de elementos que se prestan a una caracterización de naturaleza formal y de su funcionamiento.

En otro orden de cosas, las producciones de los aprendices ponen de manifiesto que las estrategias a las que recurren para reducir la complejidad del sistema verbal del español se basan en la hipótesis de 
que existen entre las formas verbales relaciones de naturaleza y alcance muy dispares y que presentan cierta tendencia a la variación hasta el momento en que se estabiliza (o se domina) el sistema. En este sentido, las producciones de los aprendices muestran que es necesario tener en cuenta tanto el sistema en su totalidad como formas verbales completas y los paradigmas que corresponden a sus lexemas respectivos, si bien sus errores sugieren que al menos algunas de las relaciones hipotéticas en las que basan sus ideas acerca de la organización interna del sistema resultan erróneas, a diferencia de lo que ocurre con los hablantes nativos. El hecho de que el alcance de las descripciones basadas en los conceptos de 'morfoma' o motivadas en los procesos de convergencia propugnados por Herce (2020) o bien en las relaciones de implicación tal como las concibe Palancar (2019) queda limitado a un fragmento más o menos amplio del sistema impide que puedan dar cuenta de manera interesante de la organización interna de ese sistema, al igual que ocurre con la presentación tradicional de los verbos, que tiende a limitarse a la mención de la forma del infinitivo. Por su parte, la propuesta de Stump y Finkel (2013) se basa en la hipótesis de la existencia de unas relaciones de implicación en las que están involucradas todas las formas que componen el paradigma de un lexema y que tienden a presentar una misma raíz (pp. 79-80). Debido a este hecho, resulta difícil de aplicar a un sistema en el que, de manera característica, se produce más variación en las raíces que en las desinencias verbales y a veces variación simultánea en la raíz y la desinencia verbal, como ocurre en español. Intentamos reflejar estas características del sistema verbal del español recurriendo a una interpretación más dinámica de las relaciones de implicación que permite aunar las aportaciones de las propuestas anteriores.

\section{2.- Formas de referencia, relaciones de implicación y predictibilidad}

Una manera de constreñir las relaciones que existen entre las formas que constituyen un paradigma flexivo consiste en determinar de manera precisa y metódica las relaciones de interdependencia que existen entre ellas, lo que implica proceder a una discriminación entre ellas sobre la base de su predictibilidad y previsibilidad. Una descripción de este tipo, más restrictiva que las que están implícitas en 
las producciones de los aprendices (cf. Finkel y Stump, 2009; Stump y Finkel, 2013), se basa en el cotejo de formas verbales completas, puesto que solo a partir de ellas (y no de los temas verbales; cf. Ambadiang, 2015) es posible obtener informaciones acerca de la configuración interna de los paradigmas verbales y de las funciones paradigmáticas en las que queda reflejada. Al existir dos subsistemas básicos dentro del sistema verbal del español y al producirse un paralelismo absoluto solo en el marco del subsistema regular, en el caso de aquellos paradigmas que incluyen formas verbales irregulares, el análisis se adaptará a la configuración paradigmática típica de cada lexema verbal puesto que tiene que determinar cuáles de sus formas contraen relaciones de implicación por un lado y, por otro, cuáles de las formas implicadas en cada relación de implicación resultan previsibles o predictibles y cuáles no en términos relativos.

Tal orden tendrá diversas motivaciones, coincidentes en parte con las propuestas reseñadas antes en el apartado 3, dependiendo de las formas involucradas en cada caso. A modo de ejemplo, una representación léxica basada en el 'morfoma L' asocia la forma de primera persona de singular del presente de indicativo a las formas del presente de subjuntivo en una relación de implicación que procede desde aquella hacia estas últimas y cuya motivación descansa no solo en cuestiones numéricas (una forma frente a seis), sino también en la impredictibilidad, manifiesta en la variación típica de series tales como miento, puedo, pongo, salgo, siento, vengo, etc. Por otra parte, las relaciones de implicación que se producen entre formas verbales están determinadas por el hecho de que las desinencias flexivas se caracterizan por una marcada estabilidad y consistencia, así como por una mayor variabilidad de las raíces en el caso de algunos lexemas verbales. De acuerdo con esta última observación, las raíces verbales adquieren una importancia muy significativa en el análisis. El proceso de segmentación que se sigue de ese cotejo permite determinar el grado de ubicuidad de una raíz verbal dentro del paradigma de su lexema. Así, al lado de relaciones que involucran formas de dos subparadigmas determinados (por ejemplo, una forma de presente de indicativo con formas del presente de subjuntivo, o bien una forma del indefinido con formas del imperfecto de subjuntivo), existen otras que tienen un 
carácter más simétrico (Ackerman, Blevins y Malouf, 2009, p.74). Es lo que ocurre, por ejemplo, con la relación que existe entre las formas siguientes de un lexema verbal del tipo de sentir: sintamos, sintáis, sintieron (sintiese, etc.), sintiendo.

Una manera de deshacer la simetría, que solo sugerimos aquí, consistiría en establecer entre las formas consideradas en cada caso un orden o una distinción sobre la base de la frecuencia (una forma más frecuente se podría considerar más básica que otra menos frecuente), la finitud (una forma conjugada se puede considerar más básica que una forma no conjugada, a menos que resulte aislada en su subparadigma, como ocurre con durmamos, muramos y sintáis por ejemplo), el tiempo y modo (una forma de indicativo se puede considerar como más básica que una de subjuntivo), el número y la persona (una forma de primera persona de singular del presente de indicativo se puede considerar más básica que cualquiera otra), aparte de la tendencia a la impredictibilidad (la primera persona de singular del presente de indicativo es la que más variaciones presenta en cuanto a la forma de la raíz y la que más casos de homonimia registra entre formas de diversos verbos (cf. siento: sentar/sentir; etc.), así como con formas no verbales.

Las observaciones aducidas en este subapartado y en el anterior son consistentes con la idea de que los diferentes elementos de un sistema flexivo están interconectados en mayor o menor medida y, por consiguiente, su estudio implica el examen de esas interconexiones. Más aún, esas observaciones justifican las relaciones implícitas en las representaciones léxicas de verbos caracterizados como irregulares que proponemos en el próximo apartado.

\section{5.- Las formas de referencia $y$ sus implicaciones para la enseñanza/aprendizaje del sistema verbal del español 5.1.- Relaciones léxicas y efectos de memorización}

Las observaciones de los apartados anteriores reflejan la disparidad de los elementos irregulares, así como el esfuerzo que hacen usuarios y, sobre todo, los aprendices del español para dar coherencia al 
sistema verbal de esta lengua, en un intento de reducir su complejidad interna o cuando menos sus efectos. Ello pasa por una reducción del número de formas verbales que figuran en la representación léxica de cada lexema verbal (que correspondería, de acuerdo con nuestra propuesta, a su entrada léxica) y que, en tanto que formas de referencia, deben ser memorizadas. Tal selección, motivada en las diversas clases de relaciones que existen entre las formas de un lexema verbal, según hemos señalado antes, constriñe la representación léxica de los lexemas verbales reduciéndola a las llamadas formas de referencia. La relación que se produce entre estas formas y las formas predecibles descansa en un proceso de memorización en el caso de las formas de referencia y de formación o generación en el de las formas predecibles. A este respecto Stump y Finkel (2013, p. 165) asocian dos clases de funciones a las formas de referencia. La primera, de naturaleza deductiva, concierne la formación de todos los elementos que conforman el paradigma de un lexema verbal dado, mientras que la segunda, enfocada en la identificación de las formas flexionadas, consiste en la asignación de alguna clase flexiva a cada una de las formas documentadas de acuerdo con sus características flexivas. En el mismo sentido, la disgregación del sistema verbal del español en dos subsistemas (regular e irregular), sugerida en el apartado 4, tiene mucho interés para la memorización, así como para la representación léxica de los verbos desde el punto de vista de las formas de referencia, cuya lista incluye al menos una forma que se consideraría como forma básica (cf. Allbright, 2008).

Una distinción que se solapa parcialmente con la anterior tiene que ver con la existencia en el sistema verbal del español de dos niveles diferenciados desde el punto de vista de la transparencia y de la canocicidad. El primero, que se podría caracterizar como sistémico (o transparadigmático; cf. Finkel y Stump, 2009, p. 53), concierne los paradigmas característicos de los verbos regulares (reflejados en los llamados modelos de conjugación). Este nivel se caracteriza por un marcado grado de canonicidad: todas las celdas del paradigma están ocupadas y casi todas las formas que las ocupan difieren entre sí, tanto desde el punto de vista de sus rasgos morfosintácticos como de sus desinencias. El segundo nivel, que se puede caracterizar como lexemático (o paradigmático), presenta una configuración interna 
diferente según el verbo considerado en cada caso o, en términos más precisos, de acuerdo con el grado de opacidad y complejidad que presenta su paradigma. Para dar cuenta de lo que hacen los usuarios y, sobre todo, los aprendices de la lengua, es necesario tener en cuenta estos dos componentes del sistema verbal del español, así como sus interrelaciones y la tensión que en muchos casos las caracteriza. Mientras que en el caso de los verbos regulares se produce un ajuste entre los dos niveles, con los llamados verbos irregulares prevalecen las especificaciones de carácter léxico y, por tanto, se producen desajustes entre ambos niveles. Las formas o paradigmas de referencia regulares sirven de modelo para el nivel sistémico en términos generales, así como para las formas regulares que aparecen dentro de algunos paradigmas junto a otras formas que son irregulares, mientras que en el paradigma de cada lexema son necesarias formas de referencia específicas cuyo número puede variar de un lexema a otro.

En contraste con una descripción formal o teórica en la que se puede hacer abstracción de las formas verbales concretas en el marco de generalizaciones que discriminan las formas regulares e irregulares, en una propuesta de carácter pedagógico es necesario proceder sobre la base de formas verbales concretas (Finkel y Stump, 2009; Stump y Finkel, 2013). En el caso del sistema verbal español, el interés de iniciar el aprendizaje con el paradigma completo de los verbos regulares tiene que ver con el hecho de que (i) permite determinar las desinencias verbales, dando cuenta de su estabilidad y especificidad, (ii) observar las diferencias que existen entre las formas de un verbo dado y determinar sus relaciones y su distribución y (iii) determinar la distribución de las raíces verbales y las relaciones que existen entre ellas. Estas informaciones intervienen de diferente modo y con diferente alcance y efecto en la representación de los verbos, según sean regulares o no. En el caso de los verbos regulares, una vez que disponemos de la información relativa a los llamados verbos modelo, en principio cualquiera de sus formas puede servir como referencia o modelo para los otros verbos de la conjugación considerada en cada caso. A modo de ejemplo, en el caso de verbos del tipo de hablar, temer o partir, formas de cita tales como las de infinitivo son suficientes para su identificación con los verbos modelo de sus conjugaciones 
respectivas $\mathrm{y}$, por tanto, para determinar las desinencias que pueden recibir en cada contexto. En tales casos, la forma de infinitivo de un verbo contiene información suficiente para deducir las formas restantes de su paradigma sobre la base del paradigma del verbo que sirve de referencia para su conjugación.

Las representaciones de los verbos irregulares, por su parte, se basan por un lado en el paradigma del verbo que sirve de referencia para su conjugación desde el punto de vista de las desinencias y, por otro, en informaciones de carácter léxico que tienen que ver con la distribución de las variantes de su raíz. Diferentes clases de relaciones se pueden establecer a este respecto (cf. RAE/ASALE, 2009 para una descripción más detallada). Por una parte, están los verbos que presentan una irregularidad que funciona de manera paralela en las formas del presente de indicativo y de subjuntivo. Es el caso con verbos del tipo de jugar, temblar, perder y poder que diptongan en las mismas personas (y en los mismos contextos morfofonológicos) en ambos subparadigmas. En otros casos, se produce un paralelismo parcial, como ocurre con verbos del tipo de dormir, pedir y sentir cuyas formas de primera y segunda formas de plural del subjuntivo presentan de manera característica el cierre de la vocal de la raíz (cf. durmamos, pidamos y sintáis). Con verbos de este tipo, como se puede observar, la forma del infinitivo no aporta informaciones suficientes para deducir las formas diptongadas, ni aquellas que se caracterizan por un cierre vocálico. De ahí la necesidad de incluir la forma de primera persona de singular del presente de indicativo en la representación.

Otras alternancias se basan en la irregularidad de la primera persona de singular del presente de indicativo y en la tercera persona de plural del indefinido. Si bien la forma de la primera persona de singular del presente de indicativo es la que entra en un mayor número de asociaciones entre formas irregulares, en la mayor parte de las ocasiones contrae una relación de implicación solo con las formas del presente de subjuntivo, como ocurre con verbos del tipo de nacer, por ejemplo. Otro tanto ocurre con verbos del tipo de caer y traer en los que se produce también la inserción de un segmento velar (/g/). Otros verbos, como poner, tener, salir y venir también insertan un segmento 
velar en la forma de primera persona de singular del indicativo, y esta irregularidad tiene alcance sobre el presente de subjuntivo, además de estar en correlación con una irregularidad del subparadigma del futuro, que consiste en el uso de raíces reducidas o cortas y la inserción de un segmento coronal $(/ \mathrm{d} /$; cf. pondré, tendré, saldré, vendré). Otras irregularidades de carácter supletivo típicas de la primera persona del singular del presente de indicativo también están en correlación con esta doble irregularidad del futuro (cf. cabré, diré, habré, sabré). Si se añaden a estos casos el de verbos como poder y querer que diptongan en el presente pero presentan un futuro irregular $\mathrm{y}$, por tanto no dependiente de la irregularidad del presente, cabe hablar de simetría (o doble implicación, en el sentido de que la irregularidad típica de las formas de primera persona del presente de indicativo y la de las formas del futuro se implican mutuamente), en lugar de una relación de implicación simple, a la hora de dar cuenta de la distribución de todas estas formas.

Por su parte, los subparadigmas del imperfecto y el futuro de subjuntivo se forman sobre la base de la tercera persona de plural del indefinido (o de la tercera persona en general), lo que motiva una relación de implicación entre una forma y un subparadigma, un poco como ocurre con la forma de primera de singular del presente de indicativo y el subparadigma de presente de subjuntivo de algunos verbos.

Por último, existen formas dobles del tipo de constreñir/constriñir; balbucear/balbucir, bendecido/bendito, concluido/concluso, confesado/confeso, imprimido/impreso. En estos casos, al igual que ocurre con verbos marcadamente irregulares como haber y ser, las irregularidades, que irán señaladas en sus representaciones léxicas respectivas, tienen un alcance mucho más local.

\section{2.- Representación léxica y congruencia sistémica}

La descripción esbozada en el apartado $4 \mathrm{y}$, sobre todo, en el subapartado anterior da cuenta de relaciones como las que se 
mencionan a continuación, en las que ' $\supset$ ' indica una relación de implicación o dependencia entre dos formas verbales, ' $\leftrightarrow$ ' una relación simétrica y ',' indica la co-presencia en un paradigma o subparadigma dado de dos formas entre las que no existe ninguna relación, más allá del hecho de formar parte de un mismo paradigma.

(3)

a. Distribución paralela completa .juego...jugamos $\leftrightarrow$ juegue...juguemos .puedo...podemos $\leftrightarrow$ pueda...podamos .tiemblo...temblamos $\leftrightarrow$ tiemble...temblemos

b. Distribución paralela parcial .duermo...dormimos $\leftrightarrow$ duerma...durmamos .pido...pedimos $\leftrightarrow$ pida...pidamos

c. Relación de implicación .basada en la primera persona de singular del presente de indicativo: caigo, hago, nazco, pongo, salgo, traigo, vengo $\supset$ presente de subjuntivo. .basada en la tercera persona de plural del indefinido: pudieron, sintieron $\supset$ imperfecto (y futuro) de subjuntivo

\section{d. Simetría} .pongo, salgo, tengo, vengo $\leftrightarrow$ pondré, saldré, tendré, vendré $\leftrightarrow$ pon, sal, ten, ven

e. Co-presencia

.puedo, quiero, sé/podré, querré, sabré

A su vez, las relaciones indicadas en (3) dan pie a representaciones léxicas, como las siguientes, que incluyen las formas de referencia de algunos paradigmas verbales que presentan formas irregulares y donde ';' indica la co-presencia en una representación de formas entre las que no existe ninguna relación más allá del hecho de ser parte del paradigma de un mismo lexema, '-' indica dobletes y ' $\varnothing$ ' indica una casilla vacía dentro del paradigma de un lexema.

a. Verbos regulares

.hablar, temer, partir (cf. cantar, comer, vivir, respectivamente) 
b. Verbos con una irregularidad .temblar; tiemblo .pedir; pido; pidieron $\leftrightarrow$ pidiendo $\leftrightarrow$ pidamos

c. Verbos con dos irregularidades .dar; doy; di .morir; muero; muramos; murieron $\leftrightarrow$ muriendo $\leftrightarrow$ muramos .sentir; siento; sintamos, sintieron $\leftrightarrow$ sintiendo $\leftrightarrow$ sintamos .salir; salgo $\leftrightarrow$ saldré $\leftrightarrow$ sal

c. Verbos con más de dos irregularidades .hacer; hago $\leftrightarrow$ haré; hicieron; haz .poder; puedo; podré; pudieron; pudiendo .poner; pongo $\leftrightarrow$ pondré; pusieron; puesto; pon .saber; sé $\leftrightarrow$ sepa $\leftrightarrow$ sabré; supieron .tener; tengo $\leftrightarrow$ tendré; tuvieron; ten .venir; vengo $\leftrightarrow$ vendré; vine; vinieron $\leftrightarrow$ viniendo; ven

d. Verbos con más de tres irregularidades .haber; he; has; hemos; haya; habré; hubieron; he .ir; voy; vas; vamos; vaya; iba; fueron; yendo; ve .decir; digo; diré; dijeron; diciendo; di .ser; soy; eres; es; somos; son; sea; era; fueron; sé

e. dobletes y dobles entradas .erguir; irguieron $\leftrightarrow$ irguiendo yergo-irgo/erguimos $\leftrightarrow$ yerga-irga/irgamos-yergamos; .balbucear-balbucir, Ø (primera persona de singular del presente de indicativo) y dobletes en el resto del paradigma .imprimir; imprimido-impreso .adecuar; adecúo-adecuo

\section{3.- Implicaciones desde un punto de vista didáctico}

La especificidad de la propuesta esbozada aquí se basa en la idea de que las relaciones que existen entre las formas flexionadas de un lexema verbal se prestan a diversas interpretaciones que inciden en la representación léxica de dicho lexema. Si dejamos de lado el hecho de formar parte de un mismo paradigma, esas relaciones pueden ser asimétricas o simétricas. En el primer caso, las formas implicadas contraen una relación de implicación en la que algunas dependen de otras, en contraste con las relaciones simétricas, que se producen entre 
formas interdependientes. Mientras que en las propuestas didácticas tradicionales el infinitivo tiende a desempeñar el papel de forma de referencia, hemos visto que aporta información suficiente para dar cuenta de todas las formas que constituyen el paradigma de un lexema verbal dado solo en el caso de los verbos regulares. Por otra parte, para dar cuenta del detalle de las diferencias entre las formas verbales, habría que tener en cuenta la frecuencia de la irregularidad en (algunas personas de) determinados números, tiempos y modos. De acuerdo con lo que se ha señalado antes, la configuración del sistema verbal español se basa en relaciones simétricas $y$, sobre todo, asimétricas, que se producen al nivel tanto sistémico como (sub)paradigmático. En el marco de ese sistema flexivo, las relaciones de implicación tienen como elementos de referencia tanto los paradigmas completos de los verbos regulares (modelo) como, potencialmente, cada una de las formas incluidas en ellos. Dentro de los paradigmas verbales, algunas formas concretas sirven de referencia o modelo para otras.

El interés de esta propuesta didáctica tiene que ver, por tanto, con el hecho de que las asimetrías que se producen entre las formas irregulares se interpretan en términos relacionales con alcance más o menos local. Esta interpretación está justificada en las relaciones de implicación que se producen entre determinadas formas verbales, aunque no se limita a ellas, como sugieren los fragmentos de representación léxica presentados antes. Si bien no podemos ofrecer un estudio detallado de esas relaciones aquí, su inclusión en el análisis permite modificar los ejercicios tradicionales centrados en la segmentación morfológica, la conjugación de verbos, el reconocimiento de formas verbales, etc., al inducir a basar todas las tareas en el cotejo de dos o más formas verbales, estando al menos una de ellas indicada en el propio enunciado del ejercicio. Uno de los efectos de este tipo de tarea tiene que ver con la toma de conciencia, por parte de los aprendices, de los diversos tipos de relaciones que pueden existir entre las formas verbales y, por consiguiente, con la discriminación de los espacios de mayor o menor canonicidad dentro tanto del sistema verbal en general como de los paradigmas de los lexemas verbales. 
En términos más específicos, la propuesta didáctica que se esboza aquí se basa en la idea de un acceso gradual al sistema verbal por parte de los aprendices. Ello supone empezar por el subsistema más canónico, a saber, el de los verbos regulares y, dentro de este, por los sub-paradigmas más básicos: presente, indefinido, imperfecto y futuro. La información relativa a estos sub-paradigmas debe, por otra parte, estar siempre al alcance y a la disposición de los alumnos en forma de tablas o fichas de referencia relativas a los verbos o las conjugaciones modelo. Sobre la base de esta información, cabe diseñar tareas cuya finalidad puede ser tanto determinar la conjugación de un verbo a partir de formas conjugadas incluidas en un ejercicio como conjugar una forma de acuerdo con otra forma verbal que sirve de modelo, cotejar formas verbales $y$ especificar las informaciones morfosintácticas asociadas a ellas y las diferencias y similitudes formales entre ellas, particularmente en el caso de pares mínimos. Por último, en lo que se refiere a los sincretismos y los dobletes, la tarea consistiría en especificar la distribución y la función de cada una de las formas implicadas, así como otras diferencias que pueden tener que ver con la frecuencia de uso o el carácter anticuado de alguna de ellas.

A su vez, esta información sirve de base y apoyo para el aprendizaje del subsistema irregular. Además de tareas del tipo de las que acabamos de mencionar, otras, más específicas de los paradigmas que incluyen formas verbales irregulares, pueden consistir en poner en relación una forma de referencia con una forma conjugada de un verbo dado o bien de diferentes verbos dentro de una misma conjugación o no, conjugar una forma a partir de una forma de referencia o bien, al revés, determinar la forma de referencia a partir de una forma conjugada, especificar la distribución de una forma verbal dada, así como los rasgos morfosintácticos asociados a ella. Otro tipo de ejercicios consistiría en la combinación de raíces léxicas con desinencias o bien se basaría en un proceso en el que los alumnos reflexionarían sobre sus errores y en la subsiguiente corrección de estos, así como en la determinación tanto de la distribución como de la función de las formas implicadas en dobletes (confesado/confeso). 
De acuerdo con estas observaciones, la manera en que procede la presentación del sistema verbal requiere un esfuerzo de memorización en relación solo con los verbos modelo y con las formas de referencia, al ser predecibles todas las formas restantes, lo que reduce el coste o esfuerzo cognitivo previsible en una presentación en la que no existen formas de referencia o en la que la única forma de referencia es la del infinitivo. En el mismo sentido, el énfasis que se pone en el cotejo de formas verbales favorece el adiestramiento de los aprendices para un tratamiento metódico y consistente de las formas verbales, sobre todo en lo que se refiere a los procesos de segmentación y recombinación. Además, es consistente con el uso de conceptos teóricos tales como los de raíz, desinencia, (sub)paradigma, conjugación, etc., cuya inclusión en la reflexión permite cierto grado de abstracción que puede ayudar a reducir el peso y la necesidad de la memorización (cf. Van Rijt, De Swart, Wijnands y Coppen, 2019).

\section{Conclusiones}

El propósito de este artículo ha sido estudiar la incidencia de la configuración interna del sistema verbal español en su uso y, sobre todo, en los procesos de enseñanza/aprendizaje. Se ha partido de la idea de que, al igual que ocurre con cualquier sistema flexivo, la organización interna del sistema verbal del español está determinada por la tensión que resulta de la co-presencia dentro de dicho sistema de formas y estructuras tanto regulares como irregulares con sus efectos tendentes a la sistematicidad o congruencia y la complejidad o asistematicidad del sistema. Si bien esa co-presencia es ubicua en el sistema verbal español, donde se manifiesta de manera más clara es en la asimetría que se produce entre, por una parte, las desinencias flexivas, estrechamente ligadas a las conjugaciones y poco sujetas a variación y, por otra, las raíces verbales que, en el caso de los llamados verbos irregulares, entrañan un grado variable de alternancia.

Las producciones tanto de los hablantes como, sobre todo, de los aprendices ponen de manifiesto los esfuerzos que hacen en el sentido de limitar los efectos de la complejidad en el sistema verbal del español. Nos hemos centrado en las relaciones de implicación e 
interdependencia que existen entre formas verbales y hemos intentado explicitarlas sobre la base de propuestas recientes en torno a la organización interna de los sistemas flexivos. Entre los factores que subyacen a las relaciones del primer tipo, destacan el carácter predecible o no de una forma y el orden de precedencia entre formas. Las formas básicas desempeñan el papel de 'formas de referencia' (no necesariamente de modelo) en relación con las formas restantes, con las que comparten la variante de la raíz (cf. tuvieron-tuviese) o bien cuyas raíces dependen de algún modo de las suyas propias, en cuanto a su forma y distribución (cf. salgo-saldré). En tanto que 'formas de referencia', las formas básicas intervienen en la representación léxica de los lexemas verbales y, en este sentido, se convierten en indicadores de la congruencia interna del sistema verbal, cuya huella puede diferir según tenga la representación una orientación pedagógica o teórica.

$\mathrm{Si}$ bien queda mucho por afinar en las representaciones propuestas, sobre todo en lo que se refiere a la interacción de la complejidad y la transparencia relativas al nivel sintagmático y paradigmático, el interés pedagógico de una presentación de este tipo tiene que ver con el hecho de que permite un proceso de enseñanza/aprendizaje escalonado de la flexión verbal que procedería sobre la base de fragmentos acotados de los paradigmas verbales, empezando por las formas típicas de los verbos identificados con los llamados modelos de conjugación y siguiendo con formas implicadas en relaciones de implicación e interdependencia cada vez más complejas. La transparencia relativa a la conjugación permite constreñir la representación léxica de los verbos regulares, reduciéndola a la sola forma del infinitivo. En el caso de los verbos irregulares prevalece una ausencia relativa de transparencia o, mejor, la opacidad interna característica de sus paradigmas respectivos, es decir, lo que estos entrañan de irregularidad, generalmente en asociación con la variación de las raíces verbales. Como hemos intentado mostrar, en ambos casos conviene basar la congruencia interna del sistema en formas verbales completas. Ello permite visualizar contrastes como el que se produce entre pares del tipo de salgo↔saldré y salo-salaré, es decir, de la interdependencia de ciertas formas y de los procesos flexivos asociados 
a ellas, algo que no ocurre con otras formas similares a ellas desde un punto de vista fonológico, pero no morfológico.

\section{Referencias bibliográficas}

Ackerman, F., y Malouf, R. (2016). Implicative relations in a word-based morphology. En A. Hippisley y G. Stump (Eds.), Cambridge handbook of morphology (pp. 297-328). Cambridge: Cambridge University Press. Doi: https://doi.org/10.1017/9781139814720.012

Ackerman, F., Blevins, J. P., y Malouf, R. (2009). Parts and wholes: patterns and relatedness in complex morphological systems and why they matter. En J. P. Blevins y J. Blevins (Eds.), Analogy in grammar: Form and acquisition (pp. 54-82). Oxford: Oxford University

Press. https://doi.org/10.1093/acprof:oso/9780199547548.003.0003

Albright, Adam (2008). Inflectional paradigms have bases too: evidence from Yiddish. En A. Bachrach y A. Nevins (Eds.), The bases of inflectional identity (pp. 271-311). Oxford: Oxford University Press.

Ambadiang, Th. (2015). Flexión verbal. En J. Gutiérrez Rexach (Coord.), Enciclopedia de lingüística hispánica, (pp. 584-594). Nueva York: Routledge, vol. 1. doi: https://doi.org/10.4324/978131571344152

Ambadiang, Th. (2017). Efectos de paradigmaticidad en la acentuación del verbo español. Loquens, 4, 2-16.

Ambadiang, Th., y Camus Bergareche, B. (2002). Morfología verbal del español como L2. En M. D. Muñoz Núñez et al. (Eds.), Actas del IV Congreso de Lingüística General (pp. 89-102). Cádiz: Servicio de Publicaciones de la Universidad de Cádiz, vol. 2.

Ambadiang, Th., Camus Bergareche, B., y García Parejo, I. (2008). Representación, procesamiento y uso en la morfología del verbo español. Verba, 35, 7-34.

Ambadiang, Th., y García Parejo, I. (1999). Organización del componente morfológico y errores de aprendizaje: el caso de la flexión verbal en inglés y español. Didáctica, 11, 55-76. 
Anderson, S. R. (2015). Dimensions of morphological complexity. En M. Baerman et al. (Eds.), Understanding and measuring morphological complexity (pp. 11-26). Oxford: Oxford University Press.

Doi:

https://doi.org/10.1093/acprof:oso/9780198723769.003.0002

Aveledo, F. (2006). El procesamiento de verbos regulares e irregulares en el español infantil: mecanismo dual vs. conexionismo. Boletín de Lingüística, 18, 5-32.

Bond, O. (2019). Canonical typology. En J. Audring y F. Masini (Eds.), The Oxford handbook of morphological theory (pp. 409434). Oxford: University Press. doi: https://doi.org/10.1093/oxfordhb/9780199668984.013.26

Brown, D., y Chumakina, M. (2013). What there might be and what there is: an introduction to canonical typology. En D. Brown, M. Chumakina y G.G. Corbett (Eds.), Canonical morphology and syntax (pp. 1-19). Oxford: Oxford University Press. doi: https://doi.org/10.1093/acprof:oso/9780199604326.003.0001

Clahsen, H., F. Aveledo, y Roca, I. (2002). The development of regular and irregular verb inflection in Spanish child language. Journal of Child Language, 29 (3), 591-622. doi: https://doi.org/10.1017/S0305000902005172

Corbett, G.G. (2007). Canonical typology, suppletion, and possible words. Language, 83 (1), 8-42.

https://doi.org/10.1353/lan.2007.0006

Corbett, G.G. (2009). Canonical inflectional classes. En F. Montermini et al. (Eds.), Selected Proceedings of the 6th Décembrettes (pp. 1-11). Somerville, MA: Cascadilla Proceedings Project.

Finkel, R., y Stump, G. (2007). Principal parts and morphological typology. Morphology, 17 (1), 39-75. doi: https://doi.org/10.1007/s11525-007-9115-9

Finkel, R., y Stump, G. (2009). Principal parts and degrees of paradigmatic transparency. En J.P. Blevins y J. Blevins (Eds.), Analogy in grammar: Form and acquisition (pp. 13-53). Oxford: Oxford University Press. doi: https://doi.org/10.1093/acprof:oso/9780199547548.003.0002 
Herce, B. (2020). Alignment of forms in Spanish verbal inflection: the gang poner, tener, venir, salir, valer as a window into the nature of paradigmatic analogy and predictability. Morphology, 30, 91115. doi: https://doi.org/10.1007/s11525-020-09352-8

Maiden, M. (2004). Morphological autonomy and diachrony. Yearbook of Morphology, 137-175. doi: https://doi.org/10.1007/1-40202900-4_6

Maiden, M. (2018). The Romance verb: Morphomic structure and diachrony. Oxford: Oxford University Press. doi: https://doi.org/10.1093/oso/9780199660216.001.0001

Oliva, J., Ignacio Serrano, J., Dolores del Castillo, M.J., y Iglesias, A. (2017). Cross-linguistic cognitive modelling of verbal morphology acquisition. Cognitive Computation, 9, 237-258. doi: https://doi.org/10.1007/s12559-017-9454-8

O'Neill, P. (2014). The morphome in constructive and abstractive models of morphology. Morphology, 24, 25-70.

https://doi.org/10.1007/s11525-014-9232-1

Palancar, E. L. (2019). Measuring the complexity of the stem alternation patterns of Spanish verbs. En Matthew Baerman et al. (Eds.), Morphological perspectives: Papers in honour of Greville G. Corbett (pp. 205-232). Edinburgh: Edinburgh University Press.

RAE/ASALE (2009) Nueva gramática de la lengua española. (cp. 4). Madrid: Espasa-Calpe, vol. 1.

Round, E. R. (2015). Rhizomorphomes, meromorphomes, and metamorphomes. En M. Baerman, D. Brown y G. G. Corbett (Eds.), Understanding and measuring morphological complexity (pp. 28-52). Oxford: Oxford University Press. doi: https://doi.org/10.1093/acprof:oso/9780198723769.003.0003

Sagot, B., y Walther, G. (2011). Non-canonical inflection: data, formalisation and complexity measures. En C. Mahlow y M., Piotrowski (Eds.), Systems and frameworks in computational morphology (pp. 23-45). Zurich: Springer. doi: https://doi.org/10.1007/978-3-642-23138-4_3

Stump, G. (2001). Inflectional Morphology. Cambridge: Cambridge University Press. doi: https://doi.org/10.1017/CBO9780511486333 
Stump, G., y Finkel, R. A. (2013). Morphological typology. From word to paradigm. Cambridge: Cambridge University Press. doi: https://doi.org/10.1017/CBO9781139248860

Stump, G., y Finkel, R. A. (2015). The complexity of inflectional systems. Linguistics Vanguard, 1 (1), 101-117. doi: https://doi.org/10.1515/lingvan-2014-1007

Van Rijt, J.H.M., De Swart, P.J.F., Wijnands, A., y Coppen, P.A. (2019). When students tackle grammatical problems: Exploring linguistic reasoning with linguistic metaconcepts in L1 grammar education. Linguistics and Education, 52, 78-88. doi: https://doi.org/10.1016/j.linged.2019.06.004 